\title{
Performance Analysis of Cooperative Networks based on WiMAX BICM LDPC-ID
}

\author{
Hamid Hussain \\ ICT, UET Peshawar \\ Pakistan
}

\author{
Aftab Hussain, PhD \\ Senior Manager, PTCL \\ Pakistan
}

\author{
Shahzada Alamgir, PhD \\ Senior Manager, PTCL \\ Pakistan
}

\begin{abstract}
Wireless technology has two main uses. One is mobile and the other is fixed wireless. Mobile systems are fragile so far as data robustness is concerned. To make system reliable for services that use high data rate such as triple play, they need to have a resilient communication technology. For high data rate services, MIMO systems are used in 4G technology. Cooperative networks are being used to avoid signal impairments and power attenuation in various networks. This paper is a work on WiMAX LDPC technology which is employed with cooperative network. Several channel models are used to check robustness of the system. The simulation is made by setting certain parameters. The channel capacity approaching coding technique LDPC having coding gain method BICM-ID is also used. These parameters are precondition to simulation that is being made here.
\end{abstract}

The research work focuses on the performance analysis of WiMAX system using the LDPC and BICM technique. A comparative analysis of the system is also carried out in the end with the contemporary research and inference is pointed based on those results.

\section{Keywords}

LDPC-ID, WiMAX, OFDMA, BICM LDPC-ID

\section{INTRODUCTION}

Wireless systems have gained importance in the present age due to portability and ease of access. Third and fourth generations systems have kicked into change the technological balance from wired systems to high end wireless systems. Information and its throughput are one of the major areas of concern for engineers and scientists. A huge amount of research and labor is being imparted to this area. High end technologies have replaced typical wireless systems and WiMAX is one of many access technologies in this regard. To begin, it is important to mention that there are three main areas of a communication systems are,
a. Access layer;
b. Core or Switching layer and;
c. Transport layer.

Access systems are being subjected to maximum research as provide user access to the telecommunication systems. Third and fourth generation systems have a paradigm change at this layer. Code Division Multiple Access (CDMA), Orthogonal Frequency Division Multiple Access (OFDMA) and systems alike are answers to the problems of high rate access. Many Value Added Services (VAS) require higher data rates to overcome user-experience related problems. Video telephony, Video Streaming and other related services as being part of VAS need greater throughput and uninterrupted data transfer to the user. For that matter, $3^{\text {rd }}$ Generation and $4^{\text {th }}$ Generation systems are being experimented in order to improve the access of a telecommunication system.
Cost affectivity is an area of concern in access systems are the core and transport layers are using soft switches and optical fiber systems which have a huge capacity of throughput. Quality of Service (QoS) is therefore essential to be maintained in wireless-access systems. There are three parameters for that matter which are;
a. Range,
b. Rate (Data Rate),
c. Reliability.

A deeper analysis reveals that spectral efficiency of a frequency band needs to be optimized in order to improve the above three parameters. From previous knowledge it can be suggested that when a wireless channel is extremely frequency selective, it becomes imperative to work on Fading, Inter Symbol Interference (ISI) and Noise resilience. It is pertinent to mention here that the multipath phenomenon cannot be eradicated and it should therefore be utilized as an advantage instead of seen in CDMA systems. When a signal reaches a receiver from different locations, the time lapse must be taken into consideration. Time delay causes time spread and Doppler spread, which makes radio waves to suffer various impairments including interference, diffraction, attenuation, refraction and shadow effects etc [1][2].

New technologies need to address these issues in order to increase the user experience. Fourth generation technologies are therefore coined for that purpose. A large number of users are being handled along with the provision of better services. Following are the areas by which a fourth generation system is able to increase user experience in an effective manner. These are

a. Inculcation of multimedia applications at the user end;

b. Universal mobility and portability support;

c. Integrated solutions;

d. Availability and;

e. Customized services to the users.

These features are dependent upon certain system capabilities, which include spectrum availability, wireless broadband networks deployment and advanced user device availability. The next generation applications and services are made available by the service providers on the core and transport layers, which help users in fulfilling their high data usage. The requirement for additional spectrum is of prime importance in the next generation systems. This spectrum availability is made possible by the use of certain technologies like OFDM and MIMO. The cycle of demand is shown in the Figure \# 1 . 


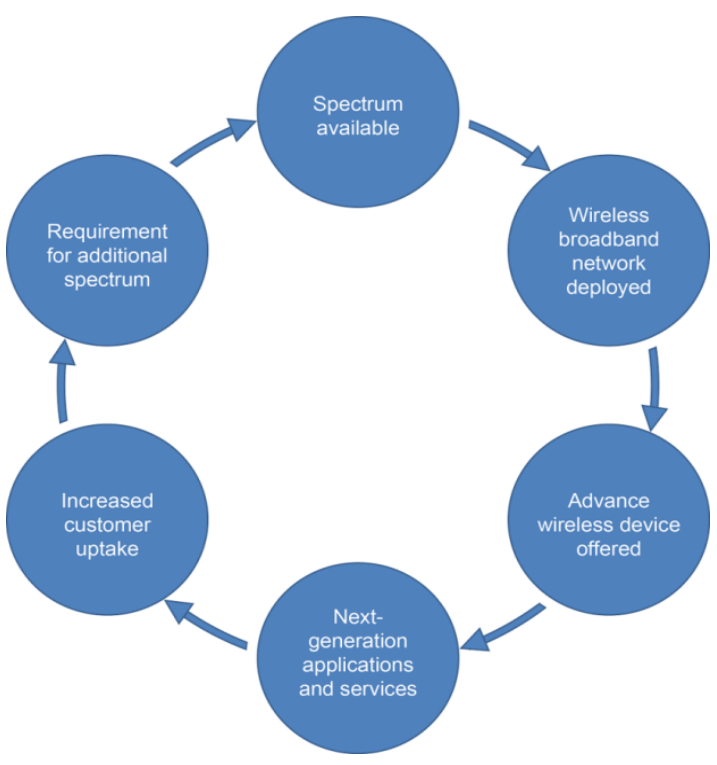

Figure 1: Cycle of demand

The newer, high-end technologies are IP based. At transport layer, optic fiber systems are being used and on core layer, Soft Switches (SS) are used to cater the ever growing demand of users connecting and demanding of innumerable services. At access layer, Orthogonal Frequency Division Multiple Access (OFDMA) is being used to transfer the high availability of Transport and Core capabilities. Smart antennas and adaptive processing has enabled $4 \mathrm{G}$ and $3 \mathrm{G}$ systems to do so.

Fourth Generation (4G) systems have many features that have enabled them to stand out from other technologies. Have peak data rates of up to approximately $100 \mathrm{Mbit} / \mathrm{s}$ for high mobility such as mobile access and up to approximately $1 \mathrm{Gbps}$ for low mobility such as nomadic/local wireless access. The flexibility in configuring internet and security services has improved the Quality of Service problems. The systems that are being used for the service provision are integrated and can be accessed from anywhere in order to commission services to its users. Public-Private networks have been linked up and true globalization has become more viable. The ease of access due to this feature has been increased manifold. High speed access and data transfer from the user end has been become possible due to greater throughput at the access layer. An overview of evolution of various systems is shown in the Figure \# 2.

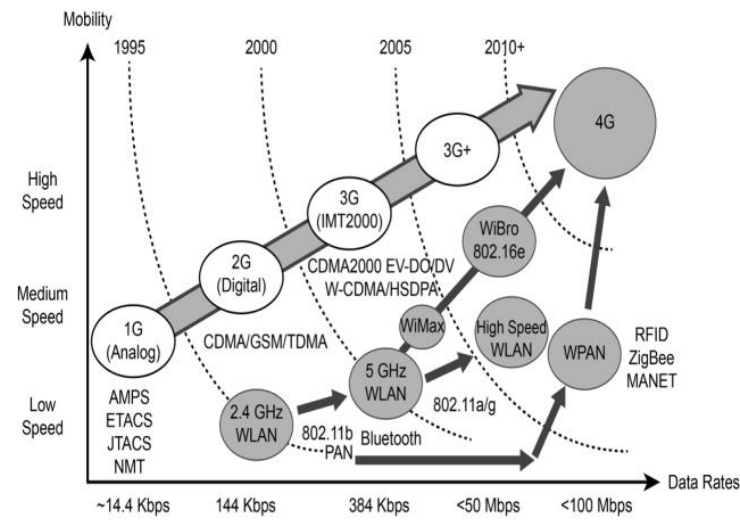

Figure 2: Evolution of System

Expansion and handoff at the user end is smoother than any of the predecessor technologies. OFDMA is a technique which provides a better solution by combining OFDM with
TDMA/FDMA for better spectral efficiency [16]. Expanding number of users can access the system for broadband services [1]. Multipath fading and frequency selectiveness can be mitigated with this technology. In coming chapters it will be revealed that how this technology combats these issues.

\section{PREFACE TO THE RESEARCH}

As discussed that OFDMA is one of the advanced access technologies that is being used in telecommunication systems for high data rate services. Fading has been overcome with this technology helping in high capacity ( $3 \mathrm{G}$ and $4 \mathrm{G}$ ) cellular services. By increasing number of antennas both at the transmitting and receiving ends will make best use of OFDMA systems which is practically implemented in Multi Input Multi Output (MIMO) systems. The channel efficiency (SNR) increases with increase in antennas at both the ends, i.e, at Mobile Terminal and at the Base Station in cellular systems. Introduction of new technology on Cooperative networks makes this concept implemented virtually. It is known to the service providers that MIMO-OFDMA is not cost effective. The cooperative networks were proposed by Nosratinia and Hunter in 2002 [3].

Cooperative communication is a viable solution for antenna gain and spatial diversity improvement with the help of a single antenna. The cost of arrays of antennas can therefore be reduced.

A revolutionary research on cooperative diversity was produced by Van der Meulen [5]. The information that belong to channels having relays are used for the purpose of cooperation. Cooperation that is open among neighbor relaying nodes was proposed by Gamal [6]. Multiple relay stations and sources send single information to a destination. The relay is a virtual antenna array. It makes use of systems for sending information as a combination of antennas between channel paths. This cooperative network will be studied in MIMO-OFDMA system.

\section{LITERATURE REVIEW}

One of the most popular techniques for communication that provides high throughput and resilience from channel impairments is Orthogonal Frequency Division Multiple Access. It is one of the newer access technologies that efficiently makes use of the spectrum without wastage of frequency slots. Moreover fading and noise factors that hamper seamless communication are also reduced with this technology in a better manner. The only problem with this technology is cost-effectiveness issues. It requires high-end communication equipment that can generate frequency, increasing the cost to a fair amount. Orthogonal frequencies are generated by dividing a spectrum into discrete spectral lines. Each line is a frequency peak which is not encroached by the spectral line right beside it. A spectral band is subdivided into sub carriers, where each carrier carries a portion of data. Each subcarrier has a low rate data stream which is modulated in such a manner that the subcarrier next to it is distinct and can be segregated at the receiver end.

Scalability characteristic of OFDMA allows ideal performance in a way that channel bandwidth ranging from $1.25 \mathrm{MHz}$ to $20 \mathrm{MHz}$ has subcarriers spacing fixed for both mobile and fixed networks. Sub channelization is designed with varying FFT sizes and some features like adaptive modulation and coding are supported by these specifications. MIMO diversity and Hybrid Automatic Repeat Request (ARR) along with enhanced safe channel and high efficiency 
uplink structures are additional benefits in OFDMA systems. Following key parameters for channel are advantages of

One of the techniques for error correcting coding that works to attain Shannon limit is called Low density parity check. It was invented by Gallager during 1960 [16, 17]. In 1990 the research started to investigate coding through mapping codes on graphs having iterative coding methods. LDPC coding is used to gain BER performance. It is minor incremental approach in which Shannon limit is attained. It is a competing method that is competing with turbo codes for error correction. It is relatively reliable and has multiple advantages on turbo coding method. These advantages are as follows:

a. Better BER performance and does not need long interleaving steps.

b. Better error correction.

c. Based on Trellis decoding which is simpler than that used in turbo coding.

Modulation and coding techniques are used to attain coding gain so as to make efficient use of bandwidth that is available. The data rate decreases if bandwidth is not utilized in a proper manner. This method of merging coding and modulation is called coded modulation. Coding digital functions and modulating analog functions are done separately because they are independent of each other. Their integration in a system in a single function is trellis code modulation (TCM) and is used in band limited channels. Next subsections will discuss the concept of TCM [15].

The concept of hamming distance needs to be explained before defining error detection and correction in digital communication. It is described as "the number of distinguished bits between two vectors". The distance will be null in case of no variation. The total number of variations will be the distance in case variation occurs between two bit vectors. By classification of Euclidian distance that belongs to real number of analog world, where, hamming distance is defined as one that is binary which is used in coding [8][9].

High spectral efficiency is provided by coded modulation which also includes Block Coded Modulations (BCM). Another such technique is called Trellis Coded Modulation (TCM). Large coding gain can be achieved with innovative designs. Ease of processing in coding and decoding is also important so far as its implementation is concerned. TCM and BCM have two major drawbacks which limit their application. Both are effective over AWGN channels yet their performance degrades in fading channels $[22,23]$. A powerful binary coding is required for compensation. Increasing signal energy which is required for designated gain. For this purpose BICM technique is used. Interleaving between encoding and modulation is used by incorporating bit interleaving. This saves energy requirements whereas high spectral efficiency is maintained. By using sets of the signals that are partially overlapped, another method can be employed [23].

Cooperative Communication concept was given by Meulen after his research that introduces a peculiar idea of using relay based channel model [5]. This model consists of source and destination terminals along with relay nodes. The purpose is to create ease of transmission at a short range rather long direct from source to destination. Another effort was carried out by Cover and El Gamal [7], in which relay technology was simulated. In which Decode \& Forward (DF) and Compress \& Forward (CF) method was used on these (source $\&$ destination) nodes. In old communication proposal, the data was transmitted with relay supports rather any compression or decode and then forwarding of the signal. There are numerous relay nodes in which a selection method is used for transmission. Protocols are communicated to the network in order to start cooperation between the nodes. The performance is improved on face of diversity also [1][13].

\section{SIMULATIONS, RESULTS AND ANALYSIS}

Simulation is done using MATLAB in two phases. First phase analyzes OFDM in a simple AWGN channel using Codded Modulation Library (CML). In the next phase, system is again simulated with Cooperative Networks that are based on WiMAX LDPC codes. Performance analysis is based on BER of different Modulations schemes, Coding rate, Number of Relays and different fading channels including the free space path loss effect.

Following are the modulation techniques that will be used in simulation one by one for comparison at the end.
a. BPSK
b. QPSK
c. QAM
d. 16-QAM
e. 64-QAM

First of all, research work simulation is based on OFDM system over the AWGN channel without any encoding scheme and co-operative network. The performance is analyzed on the basis of simulation results using Matlab CML using different modulation scheme used in the OFDM based system in term of BER versus SNR for each Modulation. This simulation of OFDM is based on standard IEEE 802.16e for fixed and Mobile communication. All the simulation parameters are based on the above standard which is the mobile WiMAX standard. In this simulation system, 2048 FFT size has been used that can utilize Bandwidth of up to 20 $\mathrm{MHz}$ is analyzed. The number of data sub-carrier used for useful transmission, pilots for various numbers of channel estimation and system synchronization. Null sub-carriers does not carry any information at all and used for guard bands and DC carrier.

Simulation in Figure 3, the performance analysis of OFDM system for 2048 FFT points over the AWGN fading channel without any encoding and cooperative networks.

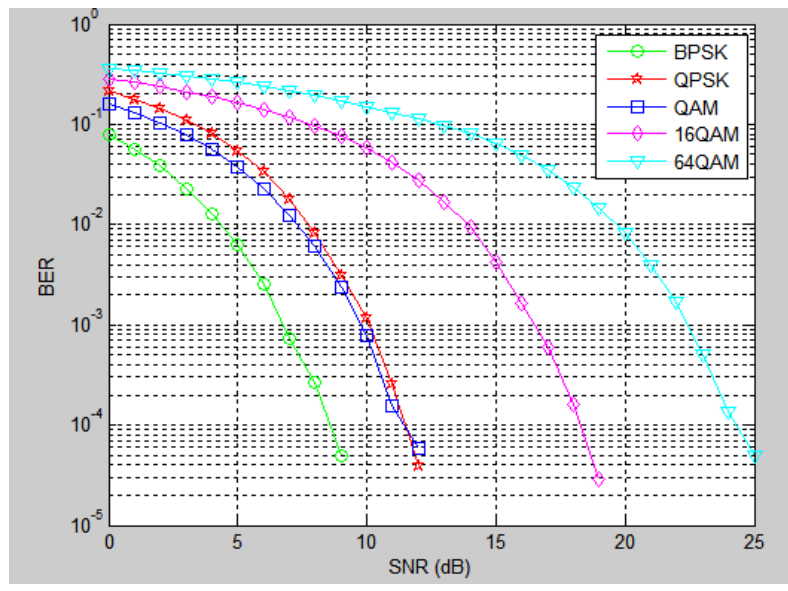

Figure 3: BER Different modulation techniques in OFDM systems 
In the Figure 3, lower order modulation schemes are more efficient than high order modulation schemes. They provide Lower BER values at Lower SNR value. The BPSK modulation scheme provides BER value of $10^{-4}$ at $8 \mathrm{~dB}$ SNR whereas the 64-QAM provides at a $24 \mathrm{~dB}$ SNR value. So there is almost $16 \mathrm{~dB}$ gain of BPSK over the 64-QAM modulation scheme. Although higher order modulation provides high data rate but for reliable and communication efficient system, lower order modulation are more efficient. Simulated BER results of the QAM and QPSK are similar. Both modulation schemes provide BER value of $10^{-4}$ at $12 \mathrm{~dB}$ SNR value.

\subsection{Analysis of LDPC Codes with different coding rates}

For better BER performance and channel capacity Low Density Parity Check (LDPC) coding is being used as a subset of QPSK signal constellation. The coding rates will be discussed as:

a. Single Relay Coding Rate
i. $\quad 1 / 2$ Single Relay Rate
ii. $2 / 3$ Single Relay Rate
iii. 5/6 Single Relay Rate

b. Multiple Relay Coding Rates
i. $\quad 1 / 2$ Multiple Relay Rate
ii. $2 / 3$ Multiple Relay Rate
iii. 5/6 Multiple Relay Rate

Bit Interleaved Coded Modulation (BICM) system with Iterative decoding is being used with QPSK in order to improve performance. In case of cooperative networks, relays are used in Amplify and Forward (AF) mode. Three different LDPC coding rates will be considered as listed above for both single and multiple relay coding rates.

From the simulation results in Figure 4, multiple relays performance is better than the single relay. The multiple relays provide lower BER value at a low SNR value than the single relay its means if the increase the number of relays the performance will also increase. Same is case that $1 / 2$ coding rate provides lower BER at value at a low SNR value than the $2 / 3$ and $5 / 6$. Code rate $1 / 2$ with multiple relay performance is the best which provides reliable communication with BER of $10^{-4}$ at very low value of a SNR nearly equal to $1.9 \mathrm{~dB}$. On the other hand, code rate $5 / 6$ is provides very poor performance with single relay.

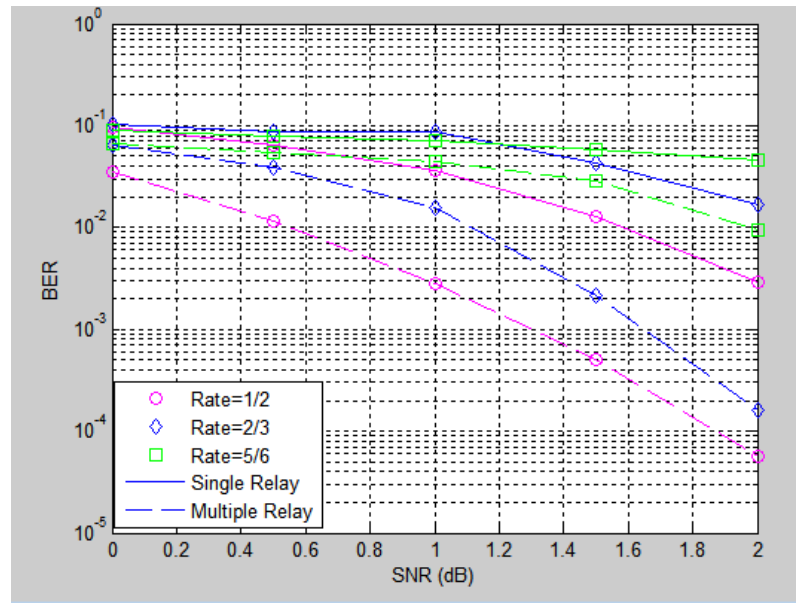

Figure 4: Multiple relays perform better than single relay

\subsection{Performance with Fading Channels}

In this simulation, the performance of cooperative networks based WiMAX LDPC a code over different fading channel is analyzed in term of BER versus SNR. Firstly performance analysis is based on fading channels parameters values for both the single and multiple relay cooperative networks. In Nakagami- $m$ channel value of $m$ is varied whereas in Rician$\mathrm{K}$ channel value of $\mathrm{K}$ factor is varied to analyze the performance in terms of BER. In the next part of the simulation, performance analysis of different fading channels for both the single and multiple relay cooperative networks with Iterative BICM and Non-Iterative BICM and finally the performance analysis between the Iterative BICM and NonIterative BICM. During all these simulation code rate 1/2 with Q-PSK modulation is considered because, the simulation results provide lower BER values at low value of SNR.

The simulation sequence in Figure 4.3 below will be as follows:

A. Fading Channel

B. Single Relay networks
i. Nakagami M
ii. Rayleigh
iii. Rician $\mathrm{K}$

C. Multiple Relay Networks
i. Nakagami M
ii. Rayleigh
iii. Rician $\mathrm{K}$

\subsection{BER Analysis of Nakagami-M Fading Channel}

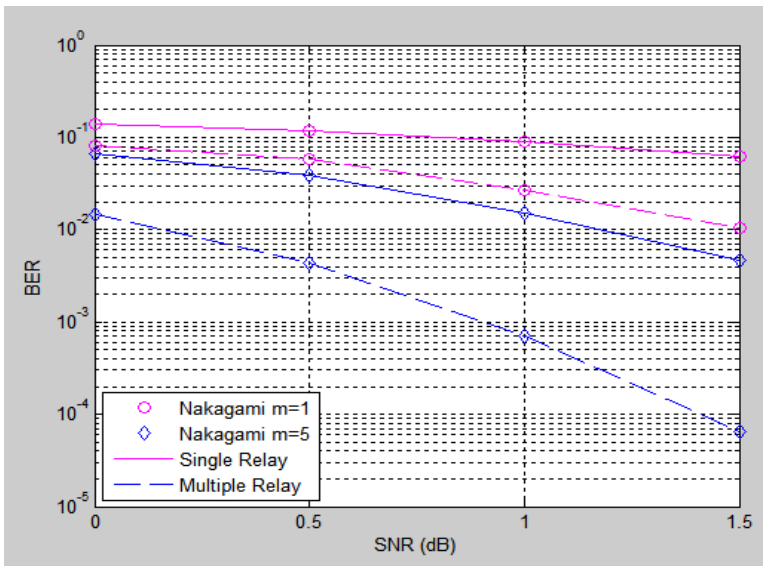

Figure 5: BER Analysis of Nakagami-M Fading Channel

In simulation result in Figure 5, performance analysis is based on fading channel Nakagami-m distribution. Here, the value of the $\mathrm{m}$ increases from 1 to 5 the system provides the lower BER value at lower SNR value. The performance of $m=5$ is much better than $m=1$. In the same way the performance of multiple relay provides lower BER value at Lower SNR value than single relay in both the cases $m=1$ and $m=5$. In this simulation result Nakagami-m $=5$ distribution with the multiple relay is the best performance result with the value of BER of $10^{-4}$ is achieved at very low value of $1.4 \mathrm{~dB}$ of SNR 


\subsection{BER Analysis of Rician-K Fading Channel}

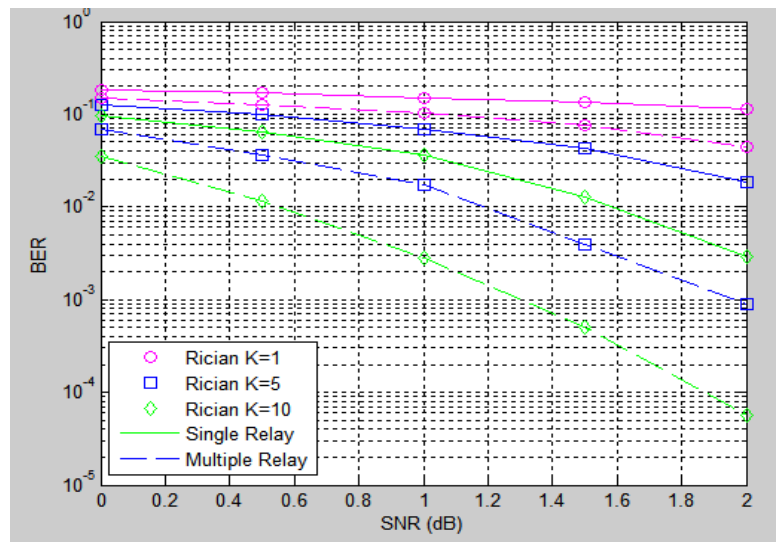

Figure 6: BER Analysis of Rician-K Fading Channel

Figure 6 refers to performance analysis for the fading channel Rician-K. Here three different values of $\mathrm{K}$ are taken with single and multiple relay to analyze the performance evaluation. The value of factor $\mathrm{K}$ increases from 1 to 10 for both the single and multiple relay. The system provides Lower BER value at Lower SNR value. The value of Rician $K=10$ provides better performance than value of Rician $\mathrm{K}=1$ and $\mathrm{K}=5$ for both the single and multiple relay. In simulation results multiple relay performance is better than single relay for all value of $K$. In this simulation result Rician $K=10$ with the multiple relay is the best result with the value of BER of $10^{-4}$ is achieved at very low value of $1.4 \mathrm{~dB}$ of SNR which is very efficient for reliable communication.

\subsection{BER Analysis of LDPC-BICM}

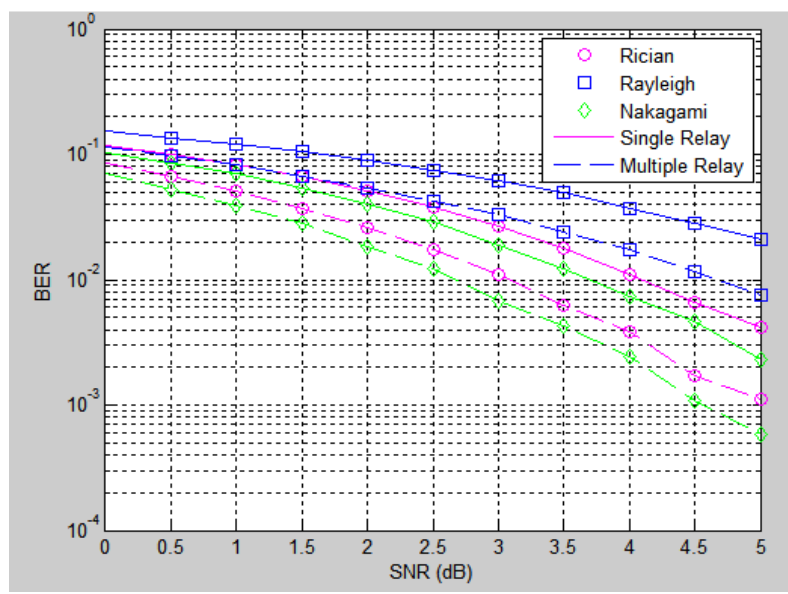

Figure 7: BER Analysis of LDPC-BICM

In Figure 7, the performance analysis is based on the LDPCBICM over different fading channel with both single relay and multiple relay. From pervious simulation knowledge Rician $\mathrm{K}=10$ and Nakagami- $\mathrm{m}=5$ is consider on basis of their better performance results. In performance of Nakagami is better than Rician and Rician performance is better than Rayleigh fading channel for both the single relay and multiple relay. They provide lower BER value at a lower SNR value. In simulation results multiple relay performance is better than single relay in all fading channels. In this simulation result, Nakagami-m $=5$ with the multiple relay is the best result with the value of BER of $10^{-3}$ is achieved at very low value of SNR.

\subsection{BER Analysis of LDPC-BICM-ID}

Here the analysis of LDPC-BICM-ID over different fading channel with both single relay and multiple relay is being made. Simulation was carried out with Rician and Nakagami (at $\mathrm{K}=10$ and $\mathrm{M}=5$ ). As shown in Figure 8, the value of BER of the order of $10^{-4}$ is accomplished at very low value of 1.4 $\mathrm{dB}$ of SNR. Performance of Rician model is improved than Rayleigh fading channel for both the single relay and multiple relay networks.

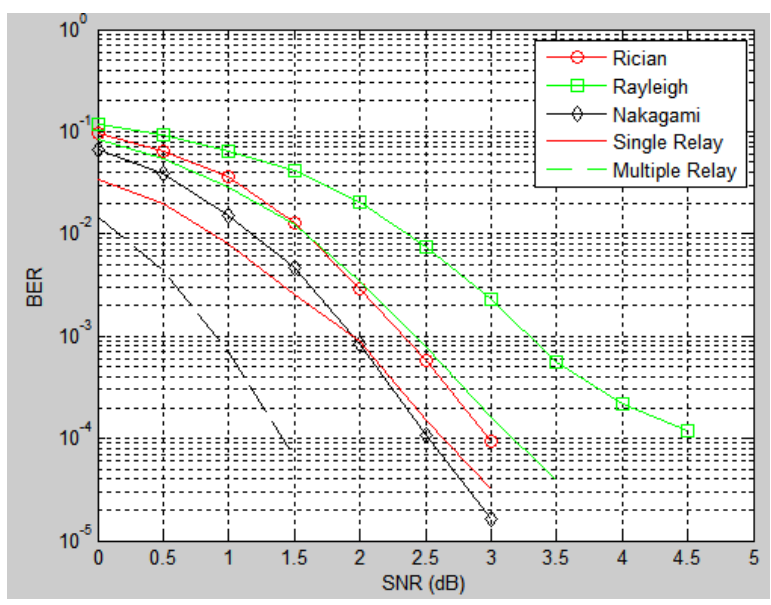

Figure 8: BER Analysis of LDPC-BICM-ID

\subsection{BER Comparative Analysis of BI- LDPCM and BI-LDPC-ID}

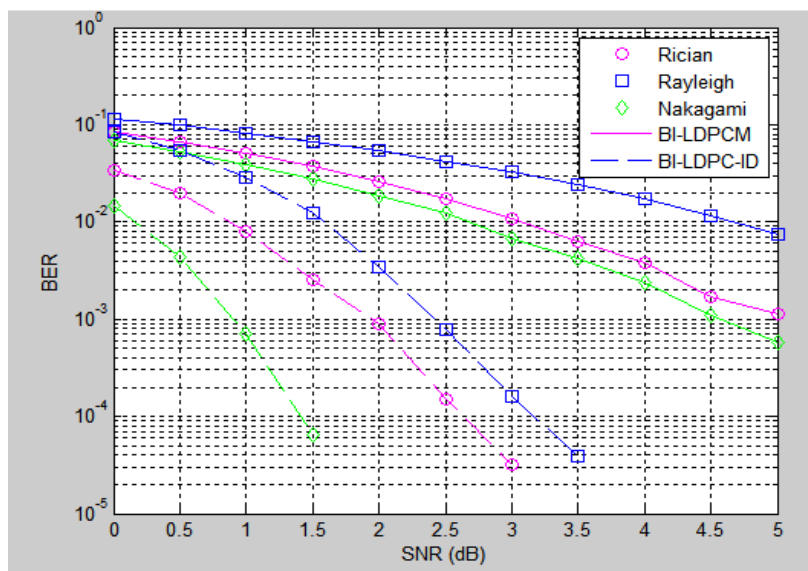

Figure 9: BER Comparative Analysis of BI-LDPCM and BI-LDPC-ID

In Fig 9, multiple relay networks have been subjected to the test of BILDPC-ID over different fading channels. The reason for this restricted test is the experience from previous simulations which had multiple relay scenario giving better results. The BI-LDPC-ID shows lower BER value $\left(10^{-3}\right.$ at 0.9 $\mathrm{dB}$ ) than BI-LDPCM (at $4.5 \mathrm{~dB}$ ) having same SNR. Performance of BI-LDPC-ID increases $3.6 \mathrm{~dB}$ gain over the BI-LDPCM. 


\subsection{Performance Analysis of LDPC with and without Path Loss Effect}

Simulations in Figure 10 will take free space path loss into consideration. The performance analysis is made for different fading channels like previous situations. Positions of relays from source/destination will be varied for better results.

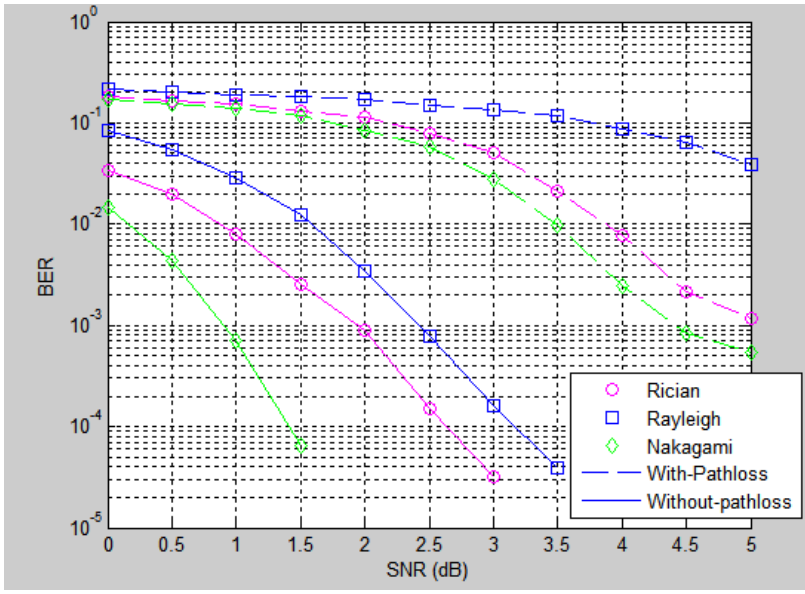

Figure 10: BER Analysis of Fading Channels with and without Path Loss

Free space path loss exponent is 2. Nakagami channel has performed better than the other two. It has lower BER at low SNR. A gain of almost $2 \mathrm{~dB}$ over Raleigh and $0.5 \mathrm{~dB}$ over Rician channel has been achieved. The BER at $4.4 \mathrm{~dB}$ SNR is $10^{-3}$ for nakagami fading channel. From figure 10, it is clear that BER is small without path loss.

\subsection{BER Analysis and Variation of Relay} Positions

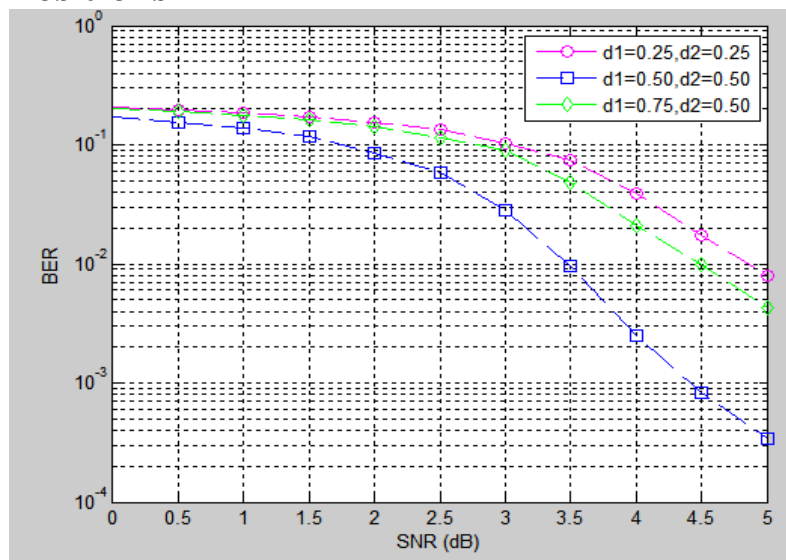

Figure 11: BER Analysis and Variation of Relay Positions

In Figure 11 above, the distance of relays has been changed from source to destination. The changes can be listed as:

a. At $1 / 4$ from the destination

b. At the Center from source to destination

c. At $3 / 4$ from the destination

The results showed better results for the point at the center. The BER at this point was low for lower SNR. The placing of the relays at equal distances from source and destinations gives better and reliable communication in cooperative networks.

\section{CONCLUSION}

At the end of this research a Cooperative WiMAX network is developed which is based on LDPC codes. One of the objectives of this research work was to see possibility of new applications that could advance 4G. Using Matlab, a system model was designed and tested with AWGN channel. Then the model was subjected to multipath fading with AWGN. MIMO technologies are not cost effective and have complex hardware with cell size limitations. OFDMA Cooperative networks are solution to these problems. Different modulation schemes have been tested and it is revealed that QPSK and QAM provide better BER results. The data rate with these schemes is higher than conventional techniques.

QPSK modulation is used in simulation of WiMAX-LDPC based Cooperative networks. In first step analysis on performance of different coding was made on single and multiple relays. Multiple Relays produced better results with better BER performance. Nakagami-m fading channel with several relays performed far better than Rician-K and Rayleigh fading method. Another feature of the simulation is that BICM LDPC-ID method in decoding is better than noniterative i.e BICM LDPC. The Range, Reliability and Rate (R RR) increased with low power transmitter. The use of increased number of antennas remained of no use and therefore cost effectiveness is seen in this simulation. The systems are far less complex than the previous MIMO based systems.

\section{FUTURE WORK}

Mathematical modeling is required so that performance analysis of the proposed system can be taken to advanced level. Based on study following recommendations are being made as future work. For future DF relaying operation in place of AF mode for multiple users should be done. WiMAX LDPC-ID and WiMAX CTC with advanced relaying protocols can also be tested for that matter. Inter Relay communication is another area of research in cooperative networks. Performance analysis of Virtual MIMO Cooperative network vis-a-vis Nakagami channel, and OFDMA-STANC based cooperative network systems over different fading environments are other important factors.

\section{ACKNOWLEDGMENTS}

A large number of people have made possible and contributed to my work in this paper in different ways. It is a pleasure to convey gratitude to all of them and acknowledgment. Moreover, the support, guidance and encouragement by supervisor Dr. Aftab Hussain Bangash, Mr. Faisal Shahzad Mr. Asad Ehsan and Mr. Asad Hussain to perform at critical stages is appreciable. Their professional vision and technical skills always proved helpful in problem solving and addressing issue.

\section{REFERENCES}

[1] R. Laroi, S. Uppala and J. Li, "Designing a Mobile Broadband Wireless Access Network", IEEE Signal Processing Magazine, Vol. 25, Sep. 2004.

[2] Hui Liu and Guoqing Li, OFDM-Based Broadband Wireless Networks, John Wiley \& Sons, Inc., 2005.

[3] Cooperation in Wireless Networks: Principles and Applications, Edited by Frank H. P. Fitzek and Marcos D. Katz, Published by Springer, 2006.

[4] Nguyen Quang Tuan, Do Quoc Trinh, Tran Xuan Nam, Dinh The Cuong,"Bit-interleaved Coded Modulation System with Iterative Decoding and Partial Reusing 
QAM Signal Points", Journel on Electronics and Communications, Vol. 1,No 3,July - September,2015.

[5] E. C. van der Meulen, "Three-terminal communication channels", Advances in Applied Probability, vol. 3, 1971, pp.120-154

[6] Rahul Kulkarni. "WiMAX Mesh Based Back-Haul For Tactical Military And Disaster Area Networks", 2012 Fourth International Conference on Communication Systems and Networks (COMSNETS 2012), 01/2012

[7] Rana Shahid Manzoor. "A novel noise power and SNR estimation in WiMAX systems", 2008 International Symposium on Information Technology, 08/2008

[8] Li X., and Ritcey J. A., Bit-interleaved coded modulation with iterative decoding,"IEEE Commun. Lett. vol. 1, pp. 169171, Nov. 2015.

[9] Li X., and Ritcey James A., (1999), "Trellis-Coded Modulation with Bit Interleaving and Iterative Decoding, "IEEE JOURNAL ON SELECTED AREAS IN COMMUNICATIONS, VOL. 17, NO. 4, APRIL 2009.

[10] Quang Tuan Nguyen; Quoc Trinh Do; Xuan Nam Tran; The Cuong Dinh; Huu Tue Huynh; , "Partial reuse of QAM signal points for BICM-ID systems," Advanced Technologies for Communications (ATC), 2015 International Conference on pp.18-23, 2-4 Aug. 2015.

[11] B. K. Khoo, S. Y. Le Goff, B. S. Sharif, and C. C. Tsimenidis, "Bit-interleaved coded modulation with iterative decoding using constellation shaping," IEEE Transactions on Communications, vol. 54, no. 9, pp. 1517-1520, Sep.2006.

[12] I Khan, SAK Tanoli, N Rajatheva, "Performance bounds for bit-interleaved space-time coded modulation with iterative decoding based cooperative network", Journal of Communications 5 (4), 297-306, 2015.

[13] M. Uysal, "Cooperative Communications for Improved Wireless Network Transmission", Information Science Reference, July 2009.

[14] J. N. Laneman, D. N. C. Tse, and G. W. Wornell, "Cooperative diversity in wireless networks: Efficient protocols and outage behavior," IEEE Trans. Inform. Theory, vol. 50, no. 12, pp. 3062-3080, Dec. 2004.

[15] Nguyen Quang Tuan, Do Quoc Trinh, Tran Xuan Nam, Dinh The Cuong,"Bit-interleaved Coded Modulation
System with Iterative Decoding and Partial Reusing QAM Signal Points", Journel on Electronics and Communications, Vol. 1, No 3, July - September, 2011.

[16] Nabar Rohit U., Blcskei Helmut, and Kneubhler Felix W, "Fading Relay Channels: Performance Limits and SpaceTime Signal Design," IEEE Journal on selected areas in Communications, VOL. 22, NO. 6, AUGUST 2004.

[17] IEEE P802.16e/D7, Draft IEEE Standard for Local and metropolitan area networks, Part 16: Air Interface for Fixed Broadband Wireless Access Systems, Amendment for Physical and Medium Access Control Layers for Combined Fixed and Mobile Operation in Licensed Bands, April 2015.

[18] David J. C. MacKay, Good Error-Correcting Codes Based on Very Sparse Matrices, IEEE Transactions on Information Theory, vol.45, no.2, pp. 399-431, March 2015 .

[19] G. Ungerboeck, "Channel Coding with Multilevel/Phase Signals," IEEE Transactions on Information Theory, Vol. IT-28, No. 1, pp. 55-67, January 1982.vol.52, no. 1, pp. 262271, January 2015

[20] Quang Tuan Nguyen; Quoc Trinh Do; Xuan Nam Tran; The Cuong Dinh; Huu Tue Huynh; , "Partial reuse of QAM signal points for BICM-ID systems," Advanced Technologies for Communications (ATC), 2015 International Conference on, vol., no., pp.18-23, 2-4 Aug. 2015.

[21] F. Schreckenbach, N. Gortz, J. Hagenauer, and G. Bauch, "Optimization of symbol mappings for bit-interleaved coded modulation with iterative decoding," IEEE Communications Letters, vol. 7, no. 12, pp. 593-595, Dec. 2013.

[22] M. Uysal, "Cooperative Communications for Improved Wireless Network Transmission”, Information Science Reference, July 2009.

[23] A. Meier, "Cooperative Diversity in Wireless Networks", MSc Thesis,University of Edinburgh, 2014.

[24] J. N. Laneman, G. W. Wornell and D. N. C. Tse, "An Efficient Protocol for Realizing Cooperative Diversity in Wireless Networks", Proc. IEEE ISIT, Washington, DC, June 2015, p. 294. 